\title{
Two Thalamic Regions Screened Using Laser Capture Microdissection with Whole Human Genome Microarray in Schizophrenia Postmortem Samples
}

\author{
Kalindi Bakshi and Eileen M. Kemether $(\mathbb{D}$ \\ Department of Psychiatry, Icahn School of Medicine at Mount Sinai, One Gustave L. Levy Place, NYC, NY 10029, USA \\ Correspondence should be addressed to Eileen M. Kemether; eileen.kemether@mssm.edu
}

Received 28 December 2019; Revised 25 March 2020; Accepted 2 April 2020; Published 1 June 2020

Academic Editor: Hugo Schnack

Copyright ( 92020 Kalindi Bakshi and Eileen M. Kemether. This is an open access article distributed under the Creative Commons Attribution License, which permits unrestricted use, distribution, and reproduction in any medium, provided the original work is properly cited.

\begin{abstract}
We used whole human genome microarray screening of highly enriched neuronal populations from two thalamic regions in postmortem samples from subjects with schizophrenia and controls to identify brain region-specific gene expression changes and possible transcriptional targets. The thalamic anterior nucleus is reciprocally connected to anterior cingulate, a schizophrenia-affected cortical region, and is also thought to be schizophrenia affected; the other thalamic region is not. Using two regions in the same subject to identify disease-relevant gene expression differences was novel and reduced intersubject heterogeneity of findings. We found gene expression differences related to miRNA-137 and other SZ-associated microRNAs, ELAVL1, BDNF, DISC-1, MECP2 and YWHAG associated findings, synapses, and receptors. Manual curation of our data may support transcription repression.
\end{abstract}

\section{Introduction}

Schizophrenia (SZ), a complex genetic disorder with no unifying conceptualization of the neuropathological or genetic correlates, is considered "a collection of neurodevelopmental disorders that involve alterations in brain circuits" [1-4]. It is a brain disorder with a heterogeneous symptom profile as well as multiple affected cellular correlates in particular thalamic and cortical circuits [5]. Various functional abnormalities impacting cognition, perception, attention, and affect are evident in persons with SZ [6].

The thalamus is a subcortical brain region comprised of numerous nuclei, many of which have reciprocal connectivity with multiple cortical regions implicated in SZ. Thus, the thalamus is thought of as a nodal link in various neural circuits [7]. As pathology in one brain region can induce both structural and functional abnormalities in either mono- or polysynaptic pathways in other brain regions, we chose to study gene expression differences of a highly enriched neuronal population from a medial tier thalamic nucleus, the anterior (principal) nucleus (AN), that has previously been determined to be a SZ-associated region $[5,8]$.

Several lines of evidence point to the involvement of the AN in SZ, including deficits of AN volume and neuronal numbers [1,9-11]. However, these losses were not consistently found $[12,13]$. The AN is reciprocally connected with cingulate cortex/paracingulate gyri; efferents of the AN target the hippocampus which then project to mammillary bodies and back to the AN [14-16]. The anterior cingulate cortex was shown to have significant gene expression changes, gray matter, and volume deficits in SZ [17-20]. Functional MRI and FDG-PET neuroimaging studies of SZ subjects have demonstrated relative decreases in blood flow and glucose utilization which has been interpreted as reduced synaptic activity in particular regions of thalamus and cortex leading to a lesser metabolic demand [5]. The thalamus is thus thought to act as a synaptic network, not passively relaying incoming signals, instead integrating hippocampal and mammillary body inputs dynamically in response to preceding neural circuit activity. The AN is considered a nodal link 
for multiple functional circuits including those subserving motivation, novelty detection, memory, and learning [21, 22]. Synaptic plasticity has been shown to be a major property underlying thalamic function in the adult brain [23, 24]. The $A N$ has been shown to have long-term synaptic modifications and plays an active role in amplifying convergent hippocampal and mammillary body inputs [24, 25]. SZrelevant changes in discrete thalamic subregions may have an effect in reciprocally connected cortical fields.

The ventral posterior lateral (VPL) nucleus is a lateral tier thalamic nucleus. Its neural circuit is comprised of spinothalamic tract fibers originating at posterolateral medulla going to VPL thalamus and terminating in the primary somatosensory cortex. Neuroimaging has not demonstrated volume loss in the lateral tier nuclei in living subjects with SZ [26]. The VPL, with one report of left hemisphere deficits in SZ [27], is not considered a SZ-associated thalamic subregion $[5,28]$, and thus was chosen as a control region for AN to study SZ-relevant gene expression defects in the thalamus.

Our aim was to identify brain region-specific gene expression changes and possible transcriptional targets in a disease associated thalamic region which may be involved in the underlying neuropathology of SZ. We used laser capture microdissection to accumulatively collect a highly enriched neuronal population for whole human genome microarray study from two thalamic regions in the same subject for comparison as well as comparison of these two regions in disease (SZ) vs. normal controls (NC). The comparison of a SZ-associated thalamic region (AN) with a largely SZ-unaffected thalamic region (VPL) in the same subject was an effort to minimize the identification of gene expression changes attributed to between-subject heterogeneity and maximize the possibility of identifying diseasespecific changes. This is the first study to our knowledge to compare a disease-impacted to a nondisease impacted thalamic region in the same subject with SZ.

\section{Methods}

2.1. Materials and Template Preparation. Postmortem brain tissue was donated by The Stanley Medical Research Institute's (SMRI) brain collection. All brain specimens were screened (SMRI) to exclude neuropathological abnormalities. The specimens were collected, with informed consent from next-of-kin, by participating medical examiners between January 1995 and June 2002 (SMRI website). The project was not human subject research and thus did not require IRB approval. The cohorts ( $n=15$ each, SZ/NC) were diagnosed using DSM-IV criteria and matched by sex, age, race, postmortem interval (PMI), affected side of the brain, and medication history (Table 1). Researchers were blinded to specimen code until the data set was received at SMRI. Samples were processed (see below) in random order to minimize systematic bias arising from sample preparation. Template preparation was conducted as previously described [29]. Byne et al. delineated VPL and reviewed EK parcellations of both AN/VPL (see [1] (Figure 1 drawing). See photograph of thionin-stained neuron (Figure 2) for the VPL delineation example.
Table 1: Demographics. Total number of subjects $(n=22)$, schizophrenia cohort $(n=11)$, and normal control cohort $(n=11)$ for anterior nucleus (AN), and ventral posterior lateral (VPL) comparisons (Supplementary Tables 1-9). Female (F), male (M), and postmortem interval (PMI). From the original cohort $(n=15$ each, SZ or NC), samples with failed neuron-capture or probe sets with low intensity values or outliers (poor RNA quality or hybridization) were excluded.

\begin{tabular}{|c|c|c|c|c|c|}
\hline Subject & Gender & $\begin{array}{c}\text { Duration of } \\
\text { disease } \\
\text { (years) }\end{array}$ & $\begin{array}{l}\text { Age at } \\
\text { death } \\
\text { (years) }\end{array}$ & $\begin{array}{l}\text { PMI } \\
\text { (hrs) }\end{array}$ & $\begin{array}{c}\text { Used for } \\
\text { comparison in } \\
\text { Supplementary } \\
\text { Table \# }\end{array}$ \\
\hline SZ & $\mathrm{F}$ & 8 & 30 & 60 & 1 \\
\hline SZ & M & 32 & 52 & 61 & $1,2,3$ \\
\hline SZ & M & 17 & 30 & 32 & 1 \\
\hline SZ & $\mathrm{F}$ & 24 & 62 & 26 & $1,2,3$ \\
\hline SZ & $\mathrm{F}$ & 45 & 60 & 40 & 1,3 \\
\hline SZ & M & 33 & 60 & 31 & $1,2,3$ \\
\hline SZ & M & 5 & 32 & 19 & 1 \\
\hline SZ & M & 27 & 44 & 50 & $1,2,3$ \\
\hline SZ & $\mathrm{F}$ & 32 & 56 & 12 & $1,2,3$ \\
\hline SZ & M & 16 & 35 & 35 & $1,2,3$ \\
\hline SZ & $\mathrm{F}$ & 24 & 49 & 38 & $1,2,3$ \\
\hline NC & M & & 52 & 28 & 2,4 \\
\hline $\mathrm{NC}$ & M & & 52 & 8 & 1 \\
\hline $\mathrm{NC}$ & M & & 52 & 22 & $1,2,4$ \\
\hline NC & M & & 53 & 28 & 1,4 \\
\hline NC & M & & 44 & 10 & $1,2,4$ \\
\hline NC & $\mathrm{F}$ & & 35 & 23 & $1,2,4$ \\
\hline NC & M & & 41 & 11 & $1,2,4$ \\
\hline NC & M & & 42 & 27 & $1,2,4$ \\
\hline NC & $\mathrm{F}$ & & 35 & 40 & 1 \\
\hline NC & $\mathrm{F}$ & & 68 & 13 & $1,2,4$ \\
\hline $\mathrm{NC}$ & $\mathrm{F}$ & & 57 & 26 & $1,2,4$ \\
\hline
\end{tabular}

2.2. Laser Capture Microdissection, RNA Isolation, and Target Preparation. Tissues were prepared for laser capture microdissection as previously described [29], using biologic grade ethanol. Total RNA was isolated in $10 \mu$ l nucleasefree water for each subject using RNeasy Micro Kit (Qiagen, Hilden, Germany) following the manufacturer's instruction. Purified RNA ( $1 \mu \mathrm{l}$ ) was subjected to quality evaluation by the Agilent Bioanalyzer pico RNA assay (Agilent Technologies, Palo Alto, DA, USA). We visually examined the electropherograms of all RNA samples, and those which showed two peaks of $18 \mathrm{~S}$ and $28 \mathrm{~S}$ ribosomal RNA (rRNA) were retained and subjected to amplification and microarray assay regardless of the degree of degradation. The Arcturus PixCell II laser capture microscope system (Mountain View, CA, USA) was used at the following settings: power 40$60 \mathrm{~mW}$, target $0.300 \mathrm{~V}$, temperature $22.4^{\circ} \mathrm{C}$, current 4.6 millihertz $(\mathrm{mH})$, repeat-laser pulse time $0.2 \mathrm{~s}$, and duration $650-750 \mu \mathrm{m}$. Approximately 3000 neurons, the largest thionin-stained cells, with nucleus and cytoplasm visible, from consecutive sections were individually captured and 


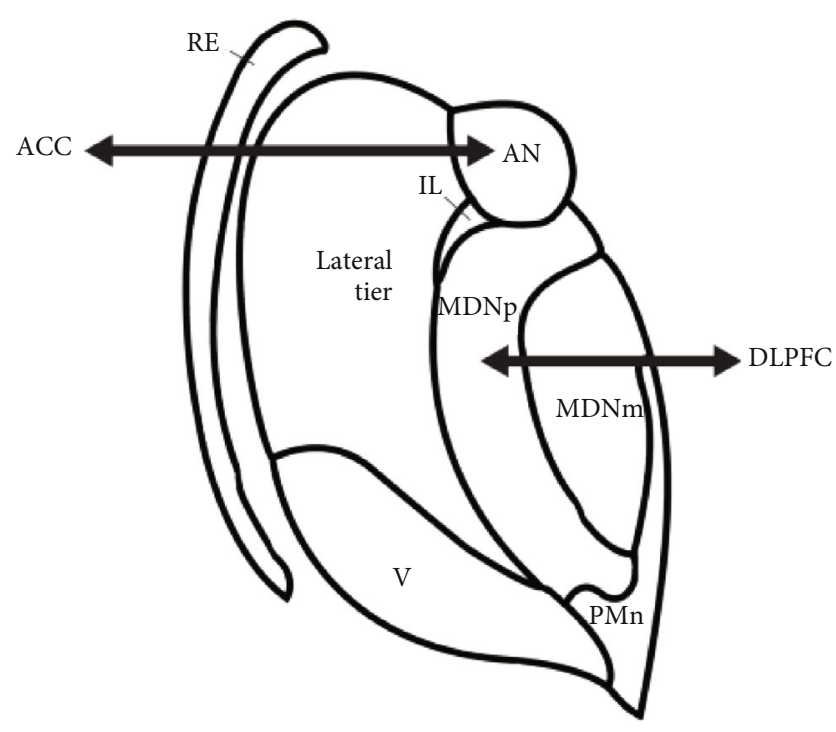

Figure 1: Thalamus drawing with subregions. Anterior (principal) nucleus (AN) reciprocally connected with the anterior cingulate cortex (ACC), mediodorsal nucleus, parvocellular subregion $(\mathrm{MDNp})$ reciprocally connected with dorsolateral prefrontal cortex (DLPFC), and ventroposterior lateral nucleus (VPL), a lateral tier nucleus.

pooled per subject. Five nanograms (ng) or maximum quantity available of qualified total RNAs were reverse transcribed and amplified using the WT Ovation Pico RNA amplification System (Nugen, San Carlos, CA, USA). The length of the amplified cDNA ranged between 50 and 2000 nucleotides, with peaks of 300-600 nucleotides from randomly sampled cDNAs assayed by Bioanalyzer.

2.3. Microarray Hybridization. Five micrograms of the single-stranded cDNAs were fragmented and end-labeled with biotin using FL-Ovation cDNA Biotin Module V2 following the manufacturer's instructions (Nugen, San Carlos, CA, USA). The whole reaction mixture of each sample was made into hybridization cocktail using the HVVS kit (Affymetrix, Santa Clara, CA, USA) following the instruction provided with the Nugen biotin labeling kit and then hybridized to Human Genome U133 Plus 2.0 Arrays (Affymetrix, Santa Clara, CA, USA). The array contains 47000 transcripts and variants covering 38500 human genes. The array images were generated through a high-resolution GeneChip Scanner 3000 7G (Affymetrix). The correlated signal intensity of spike-in controls and the percentage of present call generated based on MAS 5.0 within the GeneChip Operating Software (GCOS) were used for data quality control for each array. While our laser capture microdissection targeted neurons specifically, interneurons and incidental concurrent isolation of astrocytic foot plates, corticothalamic synapses on proximal dendrites and cell soma, as well as satellite oligodendrocytes were not completely avoidable (see Figure 3(b). in [30]. Corresponding cell-specific messenger RNAs (mRNAs) contributing to any gene expression changes in this study are considered contaminates and not an accurate reflection of disease-related changes.

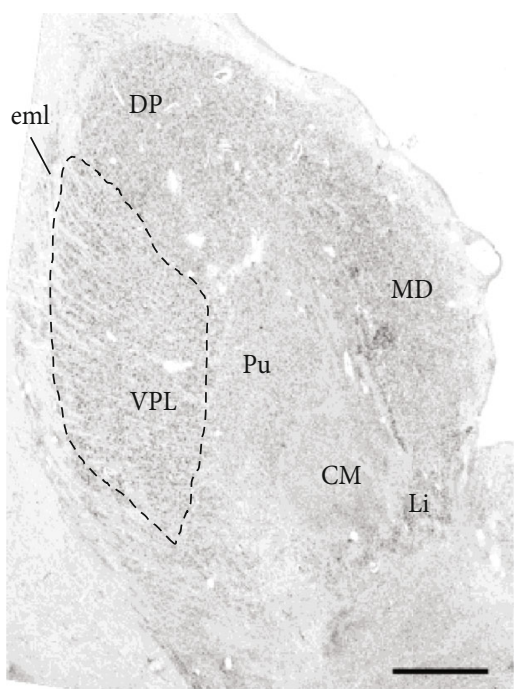

Figure 2: Postmortem Tissue Sample stained for visualization and delineation of thalamus subnuclei. Our delineation of the VPL follows that of the standardized nomenclature of Feremutsch and Simma in Dewulf [105]. We sampled from the portion of VPL in coronal sections through the caudal half of the centromedian nucleus (CM). At these levels, VPL is rather conspicuous due to its relatively larger and more coarsely staining neurons compared to surrounding nuclei. Moreover, these neurons, especially in the more lateral extent of the nucleus exhibit a prominent diagonal arrangement. The lateral boundary of the VPL is the external medullary lamina of the thalamus; its superior border, the dorsal posterior (DP) nucleus with more homogeneously arranged cells; it medial boundary is the anterior portion of the pulvinar with conspicuously smaller, paler, and more homogeneously arranged cells. Abbreviations: CM: centromedian nucleus; DP: dorsal posterior nucleus; eml: external medullary lamina; Li: nucleus limitans; MD: mediodorsal nucleus; Pu: putamen.

2.4. Microarray Data Mining and Analyses. The data were divided into disease vs. nonpsychiatric control (schizophrenia (SZ) vs. normal control (NC)) for the disease candidate gene list. From the initial $60(n=15$, each group; two brain regions, AN/VPL), a total of 38 GeneChip data were generated initially. Samples were excluded for various reasons (e.g., not enough RNA isolated, poor RNA quality or hybridization, and poor amplification). The data were analyzed by using R Bioconductor [31] statistic packages in combination with other well-known microarray and pathway analysis tools. Briefly, the raw data were first normalized across the chips by $\log$ scale robust multiarray analysis RMA [32] method, and the quality control of each chip data was performed by investigating overall intensity values for all probes, negative and positive controls. The distribution of intensity values across chips was examined, and the outliers were detected. The probe sets with low intensity values or the outliers due to poor RNA quality or hybridization were excluded.

To identify significantly differentially expressed genes between groups, the LIMMA method [33] was used to identify differentially expressed mRNAs for the following comparisons: (1) AN disease vs. AN normal control (AN 
SZ/NC) (Supplementary Table S1), (2) VPL disease vs. VPL normal control (VPL SZ/NC) (Supplementary Table S2), (3) VPL and AN disease same subject (SZ_VPL vs. AN) (Supplementary Table S3), and (4) VPL and AN normal control same subject NC_VPL vs. AN) (Supplementary Table S4). The gene expression data were also integrated with various types of up to date biological information using Database for Annotation Visualization and Integrated Discovery (DAVID) v6.7 Bioinformatics Resources [34, 35], National Institute of Allergy and Infectious Diseases (NAID), NIH (05/26/2012) http://david.abcc.ncifcrf.gov/, and Ingenuity System (http://ingenuity.com) to perform Gene Ontology (GO) function, pathway and transcription factor binding sites (TFBS) enrichment analysis, and gene/protein association network analysis for better interpretation of gene expression [27] profiles in the context of pathways, biologic function. Ingenuity Pathway Analysis (IPA) is a biological data analysis software program from Ingenuity ${ }^{\circledR}$ Systems which provides biological insight into interactions between genes, pathways, and disease processes in various experimental platforms, including microarray data. Pathway and network analysis of differentially expressed genes was performed (7/13/2015) using IPA (Ingenuity, Redwood City, CA; http://www.ingenuity.com) software licensed by Mount Sinai School of Medicine. The datasets are publicly available for download at https://www.stanleygenomics.org.

\section{Results}

A summary for the total numbers of differentially expressed transcript changes, both up- and downregulated, for each of the conditions is provided in Table 2.

3.1. Differentially Expressed Genes in the Anterior Nucleus (AN) of Schizophrenia Brains Compared with Normal Control Brains (AN SZ/NC) Fold Change 2.0, $P<0.05$. Using the LIMMA method, we compared schizophrenia $(n=11)$ vs. normal controls $(n=10)$ subjects for thalamic AN and identified differentially expressed genes (55 upregulated genes highlighted in red and 482 downregulated genes highlighted in green) listed in Table S1. IPA identified networks, pathways, and upstream regulators are listed in Tables S1.1, S1.2, and S1.3, respectively. Network molecules are related to multiple diseases and functions including hereditary, neurological disease, developmental disorders, and cellular assembly and organization. The top five pathways identified were mitochondrial dysfunction, oxidative phosphorylation, unfolded protein response, protein ubiquitination, and ketogenesis.

3.2. Differentially Expressed Genes in the Ventral Posterolateral Nucleus (VPL) of Schizophrenia Brains Compared with Normal Controls (VPL SZ/NC) Fold Change 2.0, $P<0.05$. Using the LIMMA method, we compared SZ $(n=7)$ vs. NC $(N=8)$ subjects for the thalamic VPL nucleus and identified differentially expressed genes (24 upregulated genes highlighted in red and 22 downregulated genes highlighted in green) listed in Table S2. IPA identified networks, pathways, and upstream regulators are listed in Tables S2.1,
S2.2, and S2.3, respectively. Network molecules are related to cell cycle, cell death, and cell-to-cell signaling. Three pathways were identified: BMP signaling, estrogen receptor signaling, and triacylglycerol degradation.

3.3. Differentially Expressed Genes in the Same Subject Comparing Two Different Thalamic Regions for Schizophrenia Brain Subjects (SZ_VPL vs. AN) Using Adjusted P Value < 0.05. Using the LIMMA method, we compared the two thalamic regions, VPL vs. AN, in the same subjects with SZ $(n=8)$ and identified differentially expressed genes (24 upregulated highlighted in red and 180 downregulated highlighted in green) listed in Table S3. IPA identified networks, pathways, and upstream regulators are listed in Tables S3.1, S3.2, and S3.3, respectively. Network molecules are related to various diseases and functions including tissue morphology, cell-to-cell signaling and interaction, neurological disease, and developmental disorders. Pathways identified included axonal guidance signaling, dopamineDARPP32 feedback in cAMP signaling, nNOS signaling in neurons, and synaptic long term potentiation. Manual curation of Table S3 identified gene expression changes for 14-3-3 gamma (YWHAG) NCBI interactants DDX17, SPTBN1, and SRRM2; all are downregulated. In addition, manual curation of S3.3 identified MECP2 as an upstream transcription regulator.

3.4. Differentially Expressed Genes in the Same Subject Comparing Two Thalamic Regions for Normal Control Brains (NC_VPL vs. AN) Using Adjusted P Value < 0.05 . Using the LIMMA method, we compared the two thalamic regions, VPL vs. AN, in the same NC subjects $(n=9)$ and identified differentially expressed genes (877 upregulated highlighted in red and 1,153 downregulated highlighted in green) listed in Table S4. IPA identified networks, pathways, and upstream regulators are listed in Tables S4, S4.2, and S4.3, respectively. One hundred ninety-one pathways were identified (Table S4.1).

We provide a comparison of ELAVL1 NCBI identified interactants for NC AN/VPL (Table S4) (133 up- and 113 downregulated) with SZ AN/VPL (Table S3) (22 mRNAs, one up- and 21 downregulated) gene expression changes in Table S9 (see discussion below). Five are overlapping in this comparison.

3.5. Manual Curation of our Data Section Supplementary Table 5 (S5): MicroRNA-137 Findings. In our study, microRNA-137 (miR-137 and miR-137-3p) was identified as an activated upstream regulator in (a) our AN_SZ_NC (S1.3) comparison (mir-137 4 target genes; miR-137-3p 48 target genes), (b) miR-137-3p in VPL_SZ_NC (S2.3) comparison (6 target genes) and AN_VPL_NC (S4.3) (dozens of target genes) (S5). MiR-137 was not identified as an upstream regulator in our AN_VPL_SZ comparison (S5). The only predicted activated state was identified in S1.3 (activation $z$-scores of $2.000 ; 5.280$ and $P$ value of overlap $1.68 E$ $05 ; 7.28 E-05)$. In our S2.3 findings, miR-137-3p was an upstream regulator of $A D R B 1, A S P H, C A C N B 2$ [36], CTNA3 [37], GREM1 [38], and ZEB2 (an ELAVL1 
TABLE 2: Summary of transcripts showing differential expression in each condition.

\begin{tabular}{|c|c|c|c|c|c|}
\hline Comparison & $\begin{array}{l}\text { Supplementary } \\
\text { Table }\end{array}$ & $\begin{array}{l}\text { Number of differentially } \\
\text { expressed transcripts }\end{array}$ & Hypothetical & Upregulated genes & $\begin{array}{l}\text { Downregulated } \\
\text { genes }\end{array}$ \\
\hline \multicolumn{6}{|c|}{$P<0.05$, fold change 2} \\
\hline SZ AN vs. NC AN & 1 & 528 & 9 & 55 & 482 \\
\hline SZ VPL vs. NC VPL & 2 & 40 & 6 & 24 & 22 \\
\hline \multicolumn{6}{|c|}{$P<0.05$ adj. } \\
\hline SZ AN vs. SZ VPL & 3 & 200 & 5 & 24 & 181 \\
\hline NC AN vs. NC VPL & 4 & 1,984 & 60 & 878 & 1,166 \\
\hline
\end{tabular}

interactant; see section on ELAVL1 below) (S9). In S3.3 data, miR-137 was not found. In S4.3, dozens of genes were identified as targets of miR-137 upstream regulator.

Supplementary Table 6 (S6): our data compared with [39]. Supplementary Table S7 (S7): our data compared with [40].

Supplementary Table S8 (S8): our data compared with [41]. In the schizophrenia upstream regulator comparisons, all miRNAs were activated (S1.3, S3.3), while in S2.3, of 2 miRNAs represented, neither was activated. In S4.3, of the 6 miRNAs, two were activated (miR-96-5p and miR-134).

Supplementary Table S9 (S9): MeCP2 was identified as an upstream transcription regulator in our SZ comparisons (S1.3, activation $\mathrm{Z}$ score 0.698 , Target molecules in the dataset: GRIN2A, RAB39B, HRASLS, SLC2A3, UBE3A, YWHAB); S3.3, $P$ value of overlap $3.86 E-02$, target molecules in dataset APOE, EHMT2, MBP) and control (S4.3 log ratio -0.823; activation -0.239).

ELAV-like protein 1 (ELAVL1; HuR) is one of many $\mathrm{MeCP} 2$ interactants (NCBI). Manual curation of our data identified an almost 10-fold difference in gene expression changes for ELAVL1 targets for S3 and S4 reflected in this Supplementary Table S9.

Supplementary Table S10 (S10): manual curation of our data for synapse-related genes [42] and receptors. In S1, there were 6 up- and 12 downregulated genes. In S2, there were 3 up- and 1 downregulated genes. In S3, there were 3 up- and 16 downregulated genes. In S4, there were 43 upand 92 downregulated genes.

\section{Discussion}

We compared the transcriptome of accumulatively collected neurons from a disease-impacted SZ thalamic nucleus to a nondisease thalamic nucleus in the same subject and each nucleus in SZ vs. NC (S1, S2, S3, and S4). Our discussion will focus on specific genes related to microRNA-137 (S5-7), Ingenuity Pathway Analysis microRNA findings (S8), MeCP2 interactant ELAVL1 target genes from our data (S9), and synapse-related (S10) findings. Gene expression changes identified in our SZ studies will be bolded and in our NC studies bolded and italicized.

A single-nucleotide polymorphism (SNP; rs1625579) in the microRNA-137 gene (MIR137) has been linked with SZ $[43,44]$. MicroRNA-137 expression levels were elevated in peripheral blood samples of SZ subjects who were in the ini- tial onset of the disease [45]. Manual curation of our data identified genes related to upstream regulator miR-137 (S5). In S1.3, miR-137 was an upstream regulator for GABRA1, NECAP1, NRXN1, and SPTLC1 (all downregulated; in S1.3 only). Previous reports of SZ-associated miR-137 gene targets identified in our study are ATXN1 (up-), GABRA1, GRIN2A (downregulated) (S1) and GRIN2A, NEFL (up-) and TCF4, and GRM5 (downregulated) (S4) [46, 47]. SZassociated gene ATXN1 [48] is a target of miR-137 [39, 49] (S6). It is also a protein interaction partner of ZNF804A, a target of miR-137, and 18 other SZ genes [50,51]. Transcriptional or posttranscriptional regulation of the MIR137 gene may be involved in SZ [52]. Alterations in GABRA1 $\left(\mathrm{GABA}_{\mathrm{A}}\right.$ alpha 1 receptor) subunits have been identified in SZ [49]. NRXN1 has been linked with SZ [53]. A minor copy number variation in NRNX1 may impact synapse functioning [54]. Additionally, various target genes of two SZassociated microRNAs (miR-17-5p, miR-151-3p) found previously to be dysregulated in SZ were identified (Table 3; S3.3) [55]. Other synapse-relevant microRNAs identified in our study are in Table 4 [42]. MiR-132 is necessary for neuronal functioning insofar as neuronal activity regulates its expression [56]. miR-132 was identified as a potential activated upstream regulator of various gene targets in our SZ comparisons (S1.3, S3.3), while in controls (S2.3, S4.3) it was identified but not as an activated regulator. As miR-132 is thought to impact synapses and dendritic complexity [56], our target genes may indicate thalamic synapse- and transcription-related genes in SZ.

MicroRNAs are noncoding RNAs, which can bind to messenger RNA (mRNA) to repress the expression of various gene targets [39]. MicroRNAs have various roles including transcription and translation activation and repression [57]. Each microRNA may have as many as hundreds of target mRNAs [42]. Dysregulation of one microRNA may posttranscriptionally impact the expression of thousands of genes [39]. MicroRNAs may mediate translation repression of mRNAs [41]. Since miRNA:mRNA interactions in the human brain were mapped [46], a window has been opened into the impact of miRNA and mRNA in neuronal functions. miR-137 is highly expressed in various brain regions, and it regulates adult neurogenesis, neuronal maturation, synaptic function, vesicle trafficking, and synaptic plasticity [58-61]. miR-137 is thought to target an AMPA receptor subunit and be necessary for mGluR-mediated synaptic plasticity 
TABLE 3: Schizophrenia-associated microRNAs found across independent studies (reviewed in [55]) identified in our SZ_VPL vs. AN comparison (Supplementary Table 3.3). Their targets, found in our SZ cohort, are bolded.

\begin{tabular}{|c|c|c|}
\hline $\begin{array}{l}\text { Upstream regulator in our } \\
\text { study }\end{array}$ & $\begin{array}{l}\text { SZ-associated miRNA studies } \\
\text { identified the same miRNAs }\end{array}$ & $\begin{array}{l}\text { Target molecules for micro-RNA in our study } \\
\text { (from Supplementary Table } 3(\mathrm{~S} 3) \text { ) }\end{array}$ \\
\hline $\begin{array}{l}\text { miR-17-5p (and other } \\
\text { miRNAs w/seed AAAG } \\
\text { UGC) }\end{array}$ & Santarelli et al. 2011 & $\begin{array}{c}\text { ACBD5, AHNAK, AKAP13, ANTXR1, APCDD1, ATP1A2, AUNIP, } \\
\text { CABLES1, CADM2, CELF2, CFL2, CHRM2, CMTM4, CYBB, EFCAB5, } \\
\text { ETV1, FRMD4A, FZD3, GABRA1, GDF11, GLIS3, IRF9, ITPKBLIMA1, } \\
\text { LRIG1, LRP4, MAP3K11, MAPK4, MLL, MLL3, MTSS1L, MYO10, } \\
\text { NFIB, NRP2, NTRK3, PLCB1, POLR3G, PPARA, QKI, RGMA, RHOC, } \\
\text { RYBP, SCAMP2, SDC2, SEMA4B, SOBP, SPRY4, SYT16, SYT7, } \\
\text { TENM1, TIMP2, TNRC6C, TP53INP2, TPPP, ZNF367, ZNF697, } \\
\text { ZNF704 }\end{array}$ \\
\hline $\begin{array}{l}\text { miR-151-3p (and other } \\
\text { miRNAs w/seed UAGA } \\
\text { CUG) }\end{array}$ & Gardiner et al., 2012 & $\begin{array}{l}\text { API5, CNTN2, DSCC1, ENTPD7, KIAA1217, LAPTM5, MCM6, } \\
\text { MYRIP, QKI, RERG, RGS6, RYBP, RYR3, TADA1 }\end{array}$ \\
\hline
\end{tabular}

TABLE 4: Synapse-relevant microRNAs (Gerhard [42]) found in our study.

\begin{tabular}{lcccc}
\hline Our data & \multicolumn{3}{c}{ Synapse related microRNAs identified in [42] (i.e., neuron-specific miR-124) } \\
\hline Supplementary Table 1.3 & miR-26a-5p & miR-219a-5p & & \\
AN_SZ-NC & miR132-3p & miR-219a-1-3p & \\
Supplementary Table 2.3 & miR-124-3p & miR-134-3p & & \\
VPL_SZ-NC & miR-132 & miR-219a-1-3p & & miR-219a-5p \\
Supplementary Table 3.3 & miR-9-5p & miR-134-3p & miR-124-3p & miR-124 miR-124-3p \\
AN_VPL-SZ & miR-26a-5p & miR-138-5p & miR-26a-5p \\
Supplementary Table 4.3 & miR-9 & miR-138-5p & miR-132-3p & miR-219a-5p \\
AN_VPL-NC & miR-9-5p & miR-219a-1-3p & miR-219b-3p & \\
\hline
\end{tabular}

[62]. Both receptors are implicated in the pathogenesis of SZ. miR-137 targets various mRNAs involved in glutamatergic signaling including regulating proteins in the PI3K-AktmTOR pathway which regulate response to both BDNF and neuregulin 1 signaling [41]. miR-137 may also regulate presynaptic vesicles, signals at the synapse, and plasticity of the synapse $[41,59]$. As there are multiple signaling pathways and genes in our NC IPA compared to SZ (S7), our microRNA findings may lend support to the possibility of dysregulated transcription which may impact synaptic plasticity or function in the anterior nucleus in SZ.

Evidence for DISC-1 and its interacting partners linked with SZ susceptibility is mounting [63-65]. Our study identified gene expression changes in several DISC-1 targets (CCDC136, GNB1, KIF3A, PAFAH1B1, PGK1, SRR, and TUBB) (S1) and SPTBN1, TRIO, and UTRN (S3) [66-68]. Several microRNA upstream regulators were also identified for KIF3A, PAFAH1B1, and TUBB (S8; S1.3) [41]. DISC1 target YWHAG was identified as an NMDA receptorrelated SZ de novo CNV $[67,69]$. YWHAG targets of interest in our study are DDX17 [70], SPTBN1 [71], PAFAH1B1 [72, 73], and SRRM2 [74] (S3.3). DDX17, GNB1, PRKCA, SRRM2, and TUBB (S9) are also ELAVL1 targets (see next section). Both DISC-1 and ELAVL1 interact with GNB1 (S1, downregulated). The upregulation of GNB1 was identified in the anterior cingulate cortex (ACC) of control subjects [75]. Abnormalities of DISC-1 protein may impact various proteins involved in pathways, which are vital to synapse formation, development, and maturation, including, but not limited to, glutamatergic, AMPA, and NMDA receptor complexes [66]. Our findings lend support to the dysregulation of DISC-1 and/or YWHAG-mediated signaling in SZ.

In another area, which may be of particular interest, miR137 was shown to be subject to epigenetic regulation by MeCP2 [61]. MeCP2 and a transcription factor form a complex which binds the miR-137 promoter region to inhibit transcription of miR-137 [76]. miR-137 and MECP2 have been identified as upstream regulators of various genes in our SZ cohort (see Results section Supplementary Table 9 above). Rett syndrome is a neurodevelopmental autism spectrum disorder caused by mutations, which affect germlines in any region of the X-linked MeCP2 gene [7779]. Two SNPs within the MeCP2 gene have been implicated in SZ [80, 81]. Childhood-onset schizophrenia has been linked with mutations in MECP2 [81-84]. In our study, MeCP2 was identified as an upstream transcription regulator (see Results section S9 above). While there is evidence for the involvement of de novo mutations in SZ and autism spectrum disorders [84, 85], a role for $\mathrm{MeCP} 2$ targets in $\mathrm{SZ}$ has not been shown to date. MeCP2 may regulate region- or cell-specific gene expression and is thought to have a role in the maintenance of dendritic complexity of maturing/mature neurons, maintaining excitatory synapse numbers, and in synaptic 
scaling of specific neurons [86-89]. The function of MeCP2 in specific brain regions, neuronal populations, and various cell types is becoming more defined, and there is an expression of the MeCP2e2 isoform specific to dorsal thalamus (mouse) [90-92]. If MeCP2 plays a role in the localized regulation of a subset of genes as well as chromatin maintenance in specific cells $[93,94]$, a disruption of downstream target(s) may also result in dysregulation of activity-dependent dendritic growth and/or synaptic maintenance. The specific target of MeCP2 is not yet identified in RETT to our knowledge. However, ELAV-like protein 1 (ELAVL1; HuR) is one of the many MeCP2 interactants (NCBI). It is important in the regulation and stabilization of the translation of target mRNAs $[95,96]$. Previously, two targets of ELAVL1 were identified in SZ [84]. Curiously, manual curation of our data identified an almost 10-fold difference in gene expression for ELAVL1 targets (S9) suggesting possible transcription repression in SZ. Gene expression changes of various ELAVL1 interactants in our study may reflect transcriptional dysregulation of downstream targets of ELAVL1. Our earlier transcriptome study demonstrated SZassociated gene expression differences in another thalamic region [29]. Of those 31 qPCR validated genes, 8 interact directly with ELAVL1. ELAVL1 has an important role in mRNA stability (AU-rich element mediated) and translation $[95$, 97]. ELAVL1 is localized to dendrites following experience-dependent synaptic plasticity and may have a role in neuronal mRNA posttranscriptional regulation [98100]. Posttranslational modification of ELAVL1 may regulate its ability to stabilize mRNA targets [101]. Our findings (S9) lend support to the hypothesis that ELAVL1-related genes (or absence of ELAVL1 target genes) may represent transcription repression and be disease specific for a thalamic region impacted by SZ. While MeCP2 targets have yet to be identified, we hypothesize that ineffective MeCP2 transcription regulation in the SZ cohort may occur via changes in one of its interactant targets, ELAVL1. To our knowledge, no study has identified ELAVL1 with either SZ or MeCP2.

Schizophrenia is a polygenic mental illness, with rare variants of large effect, common variants of small effect, and multiple variants of modest effect are competing for consideration in the genetic complexity of the disorder [102-104]. Our transcriptome findings are related to the previously identified GWAS miRNA-137, other SZ-associated microRNAs, and genes related to ELAVL1, BDNF, DISC-1, and YWHAG associated findings, synapses, and receptors. We hypothesize that our ELAVL1-related findings indicate transcription repression in a specific thalamic nucleus and neural circuit in SZ. To our knowledge, this study is the first to identify miR-137 as an upstream regulator of target genes identified in human postmortem tissues from schizophrenia subjects.

\section{Data Availability}

Supplementary Tables will be included with the publication.

\section{Conflicts of Interest}

The authors declare that they have no conflicts of interest.

\section{Acknowledgments}

This project was supported by Award Number R21MH078272 (EK) from the National Institute of Mental Health. The content is solely the responsibility of the authors and does not necessarily represent the official views of the National Institute of Mental Health or the National Institutes of Health. Postmortem brain tissue was donated by The Stanley Medical Research Institute Brain Collection courtesy of Drs. Michael B. Knable, E. Fuller Torrey, Maree J. Webster, and Robert H. Yolken. A special thanks to William M. Byne, M.D., Ph.D. for his oversight and advice regarding the parcellation of the thalamus and identification of VPL and AN. Thanks also to Chih-Hung Chen and Tearina T. Chu, Microarray Shared Resource Facility and Chengguo Wei, Zhengzi $\mathrm{Yi}$, and Weija Zhang, Bioinformatics, for contributions to this project.

\section{Supplementary Materials}

Supplementary Table S1: AN disease vs. AN normal control (AN SZ/NC). S1.1: Ingenuity Pathway Analysis-networks AN SZ/NC. S1.2: Ingenuity Pathway Analysis-pathways AN SZ/NC. S1.3: Ingenuity Pathway Analysis-upstream regulators AN SZ/NC. Supplementary Table S2: VPL disease vs. VPL normal control (VPL SZ/NC). S2.1: Ingenuity Pathway Analysis-networks VPL SZ/NC. S2.2: Ingenuity Pathway Analysis-pathways VPL SZ/NC. S2.3: Ingenuity Pathway Analysis-upstream regulators VPL SZ/NC. Supplementary Table S3: VPL and AN disease same subject (SZ_VPL vs. AN). S3.1: Ingenuity Pathway Analysis-networks SZ_VPL vs. AN. S3.2: Ingenuity Pathway Analysis-pathways SZ_VPL vs. AN. S3.3: Ingenuity Pathway Analysis-upstream regulators SZ_VPL vs. AN. S4: VPL and AN normal control same subject NC_VPL vs. AN). S4.1: Ingenuity Pathway Analysis-networks NC_VPL vs. AN. S4.2: Ingenuity Pathway Analysis-pathways NC_VPL vs. AN. S4.3: Ingenuity Pathway Analysis-upstream regulators NC_VPL vs. AN. S5: miR-137 comparisons in our data. S6: our data comparisons to [39] (their Table 1) Potential impact of miR-137 and its targets in schizophrenia. Front Genet Apr 26; 4:58. S7: our data comparisons to [40] Inhibition of the schizophrenia-associated microRNA-137 disrupts Nrglalpha neurodevelopmental signal transduction. Cell Rep July 5; 20(1):1-12. S8: our data comparisons to [41] microRNAs sculpt neuronal communication in a tight balance that is lost in neurological disease Front Mol Neurosci Dec 12; 11:455. S9: comparison of ELAVL1 NCBI identified interactants for NC AN/VPL (Table S4) with SZ AN/VPL (Table S3). S10: synapse and receptor related. (Supplementary Materials)

\section{References}

[1] W. Byne, S. Kidkardnee, A. Tatusov, G. Yiannoulos, M. Buchsbaum, and V. Haroutunian, "Schizophrenia-associated reduction of neuronal and oligodendrocyte numbers in the anterior principal thalamic nucleus," Schizophrenia Research, vol. 85, no. 1-3, pp. 245-253, 2006. 
[2] T. R. Insel, "Rethinking schizophrenia," Nature, vol. 468, no. 7321, pp. 187-193, 2010.

[3] H. Jaaro-Peled, A. Hayashi-Takagi, S. Seshadri, A. Kamiya, N. J. Brandon, and A. Sawa, "Neurodevelopmental mechanisms of schizophrenia: understanding disturbed postnatal brain maturation through neuregulin-1-ErbB4 and DISC1," Trends in Neurosciences, vol. 32, no. 9, pp. 485-495, 2009.

[4] T. J. Raedler, M. B. Knable, and D. R. Weinberger, "Schizophrenia as a developmental disorder of the cerebral cortex," Current Opinion in Neurobiology, vol. 8, no. 1, pp. 157-161, 1998.

[5] W. Byne, E. A. Hazlett, M. S. Buchsbaum, and E. Kemether, "The thalamus and schizophrenia: current status of research," Acta Neuropathologica, vol. 117, no. 4, pp. 347-368, 2009.

[6] J. P. Lindenmayer, P. D. Harvey, A. Khan, and B. Kirkpatrick, "Schizophrenia: measurements of psychopathology," The Psychiatric Clinics of North America, vol. 30, no. 3, pp. 339363, 2007.

[7] N. Andreasen, S. Arndt, V. Swayze et al., "Thalamic abnormalities in schizophrenia visualized through magnetic resonance image averaging," Science, vol. 266, no. 5183, pp. 294-298, 1994.

[8] T. Schallert, T. A. Jones, and M. D. Lindner, "Multilevel transneuronal degeneration after brain damage. Behavioral events and effects of anticonvulsant gamma-aminobutyric acid-related drugs," Stroke, vol. 21, 11 Suppl, pp. 143-146, 1990.

[9] W. Byne, M. S. Buchsbaum, L. A. Mattiace et al., "Postmortem assessment of thalamic nuclear volumes in subjects with schizophrenia," The American Journal of Psychiatry, vol. 159, no. 1, pp. 59-65, 2002.

[10] P. Danos, B. Baumann, H. G. Bernstein et al., "Schizophrenia and anteroventral thalamic nucleus: selective decrease of parvalbumin-immunoreactive thalamocortical projection neurons," Psychiatry Research: Neuroimaging, vol. 82, no. 1, pp. 1-10, 1998.

[11] K. A. Young, K. F. Manaye, C. L. Liang, P. B. Hicks, and D. C. German, "Reduced number of mediodorsal and anterior thalamic neurons in schizophrenia," Biological Psychiatry, vol. 47, no. 11, pp. 944-953, 2000.

[12] K. A. Dorph-Petersen, J. N. Pierri, Z. Sun, A. R. Sampson, and D. A. Lewis, "Stereological analysis of the mediodorsal thalamic nucleus in schizophrenia: volume, neuron number, and cell types," The Journal of Comparative Neurology, vol. 472, no. 4, pp. 449-462, 2004.

[13] K. A. Young, L. A. Holcomb, U. Yazdani, P. B. Hicks, and D. C. German, "Elevated neuron number in the limbic thalamus in major depression," The American Journal of Psychiatry, vol. 161, no. 7, pp. 1270-1277, 2004.

[14] J. P. Aggleton, S. D. Vann, and R. C. Saunders, "Projections from the hippocampal region to the mammillary bodies in macaque monkeys," The European Journal of Neuroscience, vol. 22, no. 10, pp. 2519-2530, 2005.

[15] R. C. Saunders, M. Mishkin, and J. P. Aggleton, "Projections from the entorhinal cortex, perirhinal cortex, presubiculum, and parasubiculum to the medial thalamus in macaque monkeys: identifying different pathways using disconnection techniques," Experimental Brain Research, vol. 167, no. 1, pp. 1-16, 2005.

[16] B. A. Vogt, D. N. Pandya, and D. L. Rosene, "Cingulate cortex of the rhesus monkey: I. Cytoarchitecture and thalamic affer- ents," The Journal of Comparative Neurology, vol. 262, no. 2, pp. 256-270, 1987.

[17] F. M. Benes, "Amygdalocortical circuitry in schizophrenia: from circuits to molecules," Neuropsychopharmacology, vol. 35, no. 1, pp. 239-257, 2010.

[18] P. Katsel, K. L. Davis, J. M. Gorman, and V. Haroutunian, "Variations in differential gene expression patterns across multiple brain regions in schizophrenia," Schizophrenia Research, vol. 77, no. 2-3, pp. 241-252, 2005.

[19] P. Roussos, P. Katsel, K. L. Davis, L. J. Siever, and V. Haroutunian, "A system-level transcriptomic analysis of schizophrenia using postmortem brain tissue samples," Archives of General Psychiatry, vol. 69, no. 12, pp. 12051213, 2012.

[20] M. Takayanagi, J. Wentz, Y. Takayanagi et al., "Reduced anterior cingulate gray matter volume and thickness in subjects with deficit schizophrenia," Schizophrenia Research, vol. 150, no. 2-3, pp. 484-490, 2013.

[21] V. van Veen and C. S. Carter, "The anterior cingulate as a conflict monitor: fMRI and ERP studies," Physiology \& Behavior, vol. 77, no. 4-5, pp. 477-482, 2002.

[22] V. Van Veen and C. S. Carter, "The timing of actionmonitoring processes in the anterior cingulate cortex," Journal of Cognitive Neuroscience, vol. 14, no. 4, pp. 593-602, 2002.

[23] J. P. Rauschecker, "Cortical control of the thalamus: topdown processing and plasticity," Nature Neuroscience, vol. 1, no. 3, pp. 179-180, 1998.

[24] M. Tsanov, S. D. Vann, J. T. Erichsen, N. Wright, J. P. Aggleton, and S. M. O'Mara, "Differential regulation of synaptic plasticity of the hippocampal and the hypothalamic inputs to the anterior thalamus," Hippocampus, vol. 21, no. 1, pp. 1-8, 2011.

[25] S. D. Vann and J. P. Aggleton, "The mammillary bodies: two memory systems in one?," Nature Reviews. Neuroscience, vol. 5, no. 1, pp. 35-44, 2004.

[26] E. M. Kemether, M. S. Buchsbaum, W. Byne et al., "Magnetic resonance imaging of mediodorsal, pulvinar, and centromedian nuclei of the thalamus in patients with schizophrenia," Archives of General Psychiatry, vol. 60, no. 10, pp. 983-991, 2003.

[27] P. Danos, B. Baumann, A. Krämer et al., "Volumes of association thalamic nuclei in schizophrenia: a postmortem study," Schizophrenia Research, vol. 60, no. 2-3, pp. 141155, 2003.

[28] E. A. Hazlett, M. S. Buchsbaum, E. Kemether et al., “Abnormal glucose metabolism in the mediodorsal nucleus of the thalamus in schizophrenia," The American Journal of Psychiatry, vol. 161, no. 2, pp. 305-314, 2004.

[29] T. T. Chu, Y. Liu, and E. Kemether, "Thalamic transcriptome screening in three psychiatric states," Journal of Human Genetics, vol. 54, no. 11, pp. 665-675, 2009.

[30] W. Byne, S. Dracheva, B. Chin, J. M. Schmeidler, K. L. Davis, and V. Haroutunian, "Schizophrenia and sex associated differences in the expression of neuronal and oligodendrocytespecific genes in individual thalamic nuclei," Schizophrenia Research, vol. 98, no. 1-3, pp. 118-128, 2008.

[31] R. C. Gentleman, V. J. Carey, D. M. Bates et al., "Bioconductor: open software development for computational biology and bioinformatics," Genome Biology, vol. 5, no. 10, p. R80, 2004. 
[32] R. A. Irizarry, B. M. Bolstad, F. Collin, L. M. Cope, B. Hobbs, and T. P. Speed, "Summaries of Affymetrix GeneChip probe level data," Nucleic Acids Research, vol. 31, no. 4, article e15, pp. 15e-115, 2003.

[33] G. K. Smyth, "Linear models and empirical bayes methods for assessing differential expression in microarray experiments," Statistical Applications in Genetics and Molecular Biology, vol. 3, no. 1, pp. 1-25, 2004.

[34] G. Dennis Jr., B. T. Sherman, D. A. Hosack et al., "DAVID: database for annotation, visualization, and integrated discovery," Genome Biology, vol. 4, no. 5, 2003.

[35] D. W. Huang, B. T. Sherman, and R. A. Lempicki, "Systematic and integrative analysis of large gene lists using DAVID bioinformatics resources," Nature Protocols, vol. 4, no. 1, pp. 44-57, 2009.

[36] Cross-Disorder Group of the Psychiatric Genomics Consortium, "Identification of risk loci with shared effects on five major psychiatric disorders: a genome-wide analysis," Lancet, vol. 381, no. 9875, pp. 1371-1379, 2013.

[37] J. Grove, A. D. Borglum, and B. D. Pearce, "GWAS, cytomegalovirus infection, and schizophrenia," Current Behavioral Neuroscience Reports, vol. 1, no. 4, pp. 215-223, 2014.

[38] Z. Liu, M. Osipovitch, A. Benraiss et al., "Dysregulated glial differentiation in schizophrenia may be relieved by suppression of SMAD4- and REST-dependent signaling," Cell Reports, vol. 27, no. 13, pp. 3832-3843.e6, 2019, e3836.

[39] C. Wright, J. A. Turner, V. D. Calhoun, and N. PerroneBizzozero, "Potential impact of miR-137 and its targets in schizophrenia," Frontiers in Genetics, vol. 4, p. 58, 2013.

[40] K. T. Thomas, B. R. Anderson, N. Shah et al., "Inhibition of the schizophrenia-associated microRNA miR-137 disrupts Nrg1 $\alpha$ neurodevelopmental signal transduction," Cell Reports, vol. 20, no. 1, pp. 1-12, 2017.

[41] K. T. Thomas, C. Gross, and G. J. Bassell, "MicroRNAs sculpt neuronal communication in a tight balance that is lost in neurological disease," Frontiers in Molecular Neuroscience, vol. 11, 2018.

[42] G. Schratt, "MicroRNAs at the synapse," Nature Reviews Neuroscience, vol. 10, no. 12, pp. 842-849, 2009.

[43] The Schizophrenia Psychiatric Genome-Wide Association Study (GWAS) Consortium, "Genome-wide association study identifies five new schizophrenia loci," Nature Genetics, vol. 43, no. 10, pp. 969-976, 2011.

[44] S. Ripke, C. O'Dushlaine, K. Chambert et al., "Genome-wide association analysis identifies 13 new risk loci for schizophrenia," Nature Genetics, vol. 45, no. 10, pp. 1150-1159, 2013.

[45] S. Wu, R. Zhang, F. Nie et al., "MicroRNA-137 inhibits EFNB2 expression affected by a genetic variant and is expressed aberrantly in peripheral blood of schizophrenia patients," eBioMedicine, vol. 12, pp. 133-142, 2016.

[46] R. L. Boudreau, P. Jiang, B. L. Gilmore et al., "Transcriptomewide discovery of microRNA binding sites in human brain," Neuron, vol. 81, no. 2, pp. 294-305, 2014.

[47] C. Wright, V. D. Calhoun, S. Ehrlich, L. Wang, J. A. Turner, and N. I. Bizzozero, "Meta gene set enrichment analyses link miR-137-regulated pathways with schizophrenia risk," Frontiers in Genetics, vol. 6, p. 147, 2015.

[48] J. Liu and B. Su, "Integrated analysis supports ATXN1 as a schizophrenia risk gene," Schizophrenia Research, vol. 195, pp. 298-305, 2018.
[49] S. Wang, S. D. Detera-Wadleigh, H. Coon et al., "Evidence of linkage disequilibrium between schizophrenia and the SCa1 CAG repeat on chromosome 6p23," American Journal of Human Genetics, vol. 59, no. 3, pp. 731-736, 1996.

[50] A. H. Kim, E. K. Parker, V. Williamson, G. O. McMichael, A. H. Fanous, and V. I. Vladimirov, "Experimental validation of candidate schizophrenia gene ZNF804A as target for hsamiR-137," Schizophrenia Research, vol. 141, no. 1, pp. 6064, 2012.

[51] H. J. Williams, Molecular Genetics of Schizophrenia Collaboration (MGS) International Schizophrenia Consortium (ISC), SGENE-plus, GROUP, N. Norton et al., "Fine mapping ofZNF804A and genome-wide significant evidence for its involvement in schizophrenia and bipolar disorder," Molecular Psychiatry, vol. 16, no. 4, pp. 429-441, 2011.

[52] A. Warburton, G. Breen, V. J. Bubb, and J. P. Quinn, “A GWAS SNP for schizophrenia is linked to the internal MIR137 promoter and supports differential allele-specific expression," Schizophrenia Bulletin, vol. 42, no. 4, pp. $1003-$ 1008, 2016.

[53] G. Kirov, D. Gumus, W. Chen et al., "Comparative genome hybridization suggests a role for NRXN1 and APBA2 in schizophrenia," Human Molecular Genetics, vol. 17, no. 3, pp. 458-465, 2007.

[54] L. Coelewij and D. Curtis, "Mini-review: update on the genetics of schizophrenia," Annals of Human Genetics, vol. 82, no. 5, pp. 239-243, 2018.

[55] N. J. Beveridge and M. J. Cairns, "MicroRNA dysregulation in schizophrenia," Neurobiology of Disease, vol. 46, no. 2, pp. 263-271, 2012.

[56] K. Sakamoto and J. J. Crowley, "A comprehensive review of the genetic and biological evidence supports a role for microRNA-137 in the etiology of schizophrenia," American Journal of Medical Genetics. Part B, Neuropsychiatric Genetics, vol. 177, no. 2, pp. 242-256, 2018.

[57] M. Strazisar, S. Cammaerts, K. van der Ven et al., "MIR137 variants identified in psychiatric patients affect synaptogenesis and neuronal transmission gene sets," Molecular Psychiatry, vol. 20, no. 4, pp. 472-481, 2015.

[58] I. Guella, A. Sequeira, B. Rollins et al., "Analysis of miR-137 expression and rs1625579 in dorsolateral prefrontal cortex," Journal of Psychiatric Research, vol. 47, no. 9, pp. 12151221, 2013.

[59] E. He, M. A. G. Lozano, S. Stringer et al., "MIR137 schizophrenia-associated locus controls synaptic function by regulating synaptogenesis, synapse maturation and synaptic transmission," Human Molecular Genetics, vol. 27, no. 11, pp. 1879-1891, 2018.

[60] S. Siegert, J. Seo, E. J. Kwon et al., "The schizophrenia risk gene product miR-137 alters presynaptic plasticity," Nature Neuroscience, vol. 18, no. 7, pp. 1008-1016, 2015.

[61] K. E. Szulwach, X. Li, R. D. Smrt et al., "Cross talk between microRNA and epigenetic regulation in adult neurogenesis," The Journal of Cell Biology, vol. 189, no. 1, pp. 127-141, 2010.

[62] N. F. M. Olde Loohuis, W. Ba, P. H. Stoerchel et al., "MicroRNA-137 controls AMPA-receptor-mediated transmission and mGluR-dependent LTD," Cell Reports, vol. 11, no. 12, pp. 1876-1884, 2015.

[63] D. T. Balu and J. T. Coyle, "Neuroplasticity signaling pathways linked to the pathophysiology of schizophrenia," 
Neuroscience and Biobehavioral Reviews, vol. 35, no. 3, pp. 848-870, 2011.

[64] L. N. Moens, P. de Rijk, J. Reumers et al., "Sequencing of DISC1 pathway genes reveals increased burden of rare missense variants in schizophrenia patients from a northern Swedish population," PLoS One, vol. 6, no. 8, article e23450, 2011.

[65] K. K. Nicodemus, J. H. Callicott, R. G. Higier et al., "Evidence of statistical epistasis between DISC1, CIT and NDEL1 impacting risk for schizophrenia: biological validation with functional neuroimaging," Human Genetics, vol. 127, no. 4, pp. 441-452, 2010.

[66] L. M. Camargo, V. Collura, J. C. Rain et al., "Disrupted in schizophrenia 1 interactome: evidence for the close connectivity of risk genes and a potential synaptic basis for schizophrenia," Molecular Psychiatry, vol. 12, no. 1, pp. 74-86, 2007.

[67] G. Kirov, A. J. Pocklington, P. Holmans et al., "De novo CNV analysis implicates specific abnormalities of postsynaptic signalling complexes in the pathogenesis of schizophrenia," Molecular Psychiatry, vol. 17, no. 2, pp. 142-153, 2012.

[68] D. Martins-de-Souza, G. Maccarrone, S. Reckow, P. Falkai, A. Schmitt, and C. W. Turck, "Shotgun mass spectrometry analysis of the human thalamus proteome," Journal of Separation Science, vol. 32, no. 8, pp. 1231-1236, 2009.

[69] Y. Ozeki, T. Tomoda, J. Kleiderlein et al., "Disrupted-inschizophrenia-1 (DISC-1): mutant truncation prevents binding to NudE-like (NUDEL) and inhibits neurite outgrowth," Proceedings of the National Academy of Sciences of the United States of America, vol. 100, no. 1, pp. 289-294, 2003.

[70] S. Mallon, B. T. Wakim, and B. Roizman, "Use of biotinylated plasmid DNA as a surrogate for HSV DNA to identify proteins that repress or activate viral gene expression," Proceedings of the National Academy of Sciences of the United States of America, vol. 109, no. 51, pp. E3549-E3557, 2012.

[71] H. J. Lee, K. Lee, and H. Im, " $\alpha$-Synuclein modulates neurite outgrowth by interacting with SPTBN1," Biochemical and Biophysical Research Communications, vol. 424, no. 3, pp. 497-502, 2012.

[72] B. K. Lipska, T. Peters, T. M. Hyde et al., "Expression of DISC1 binding partners is reduced in schizophrenia and associated with DISC1 SNPs," Human Molecular Genetics, vol. 15, no. 8, pp. 1245-1258, 2006.

[73] H. M. Moon and A. Wynshaw-Boris, "Cytoskeleton in action: lissencephaly, a neuronal migration disorder," Wiley Interdisciplinary Reviews: Developmental Biology, vol. 2, no. 2, pp. 229-245, 2013.

[74] J. Jin, F. D. Smith, C. Stark et al., "Proteomic, functional, and domain-based analysis of in vivo 14-3-3 binding proteins involved in cytoskeletal regulation and cellular organization," Current Biology, vol. 14, no. 16, pp. 1436-1450, 2004.

[75] D. Martins-de-Souza, A. Schmitt, R. Roder et al., "Sex-specific proteome differences in the anterior cingulate cortex of schizophrenia," Journal of Psychiatric Research, vol. 44, no. 14, pp. 989-991, 2010.

[76] J. Yin, J. Lin, X. Luo et al., "miR-137: a new player in schizophrenia," International Journal of Molecular Sciences, vol. 15, no. 2, pp. 3262-3271, 2014.

[77] R. E. Amir, I. B. Van den Veyver, M. Wan, C. Q. Tran, U. Francke, and H. Y. Zoghbi, "Rett syndrome is caused by mutations in X-linked MECP2, encoding methyl-CpG- binding protein 2," Nature Genetics, vol. 23, no. 2, pp. 185$188,1999$.

[78] M. Chahrour and H. Y. Zoghbi, "The story of Rett syndrome: from clinic to neurobiology," Neuron, vol. 56, no. 3, pp. 422437, 2007.

[79] E. S. Na and L. M. Monteggia, "The role of MeCP2 in CNS development and function," Hormones and Behavior, vol. 59, no. 3, pp. 364-368, 2011.

[80] T. I. Sheikh, R. Harripaul, M. Ayub, and J. B. Vincent, "MeCP2 AT-Hook1 mutations in patients with intellectual disability and/or schizophrenia disrupt DNA binding and chromatin compaction in vitro," Human Mutation, vol. 39, no. 5, pp. 717-728, 2018.

[81] E. H. M. Wong, H. C. So, M. Li et al., "Common variants on Xq28 conferring risk of schizophrenia in Han Chinese," Schizophrenia Bulletin, vol. 40, no. 4, pp. 777-786, 2014.

[82] D. Cohen, G. Lazar, P. Couvert et al., "MECP2 mutation in a boy with language disorder and schizophrenia," American Journal of Psychiatry, vol. 159, no. 1, pp. 148-149, 2002.

[83] P. Moretti and H. Y. Zoghbi, "MeCP2 dysfunction in Rett syndrome and related disorders," Current Opinion in Genetics \& Development, vol. 16, no. 3, pp. 276-281, 2006.

[84] A. Piton, J. Gauthier, F. F. Hamdan et al., "Systematic resequencing of X-chromosome synaptic genes in autism spectrum disorder and schizophrenia," Molecular Psychiatry, vol. 16, no. 8, pp. 867-880, 2011.

[85] S. E. McCarthy, J. Gillis, M. Kramer et al., "De novo mutations in schizophrenia implicate chromatin remodeling and support a genetic overlap with autism and intellectual disability," Molecular Psychiatry, vol. 19, no. 6, pp. 652-658, 2014.

[86] M. P. Blackman, B. Djukic, S. B. Nelson, and G. G. Turrigiano, "A critical and cell-autonomous role for MeCP2 in synaptic scaling up," The Journal of Neuroscience, vol. 32, no. 39, pp. 13529-13536, 2012.

[87] M. Mellen, P. Ayata, S. Dewell, S. Kriaucionis, and N. Heintz, "MeCP2 binds to $5 \mathrm{hmC}$ enriched within active genes and accessible chromatin in the nervous system," Cell, vol. 151, no. 7, pp. 1417-1430, 2012.

[88] E. S. Na, E. D. Nelson, E. T. Kavalali, and L. M. Monteggia, "The impact of MeCP2 loss- or gain-of-function on synaptic plasticity," Neuropsychopharmacology, vol. 38, no. 1, pp. 212219, 2013.

[89] Y. T. Zhao, D. Goffin, B. S. Johnson, and Z. Zhou, "Loss of $\mathrm{MeCP} 2$ function is associated with distinct gene expression changes in the striatum," Neurobiology of Disease, vol. 59, pp. 257-266, 2013.

[90] B. Lipi, L. Jaldeep, and P. Prakash, "Role of astrocytic MeCP2 in regulation of CNS myelination by affecting oligodendrocyte and neuronal physiology and axo-glial interactions," Experimental Brain Research, vol. 236, no. 11, pp. 30153027, 2018.

[91] C. O. Olson, R. M. Zachariah, C. D. Ezeonwuka, V. R. B. Liyanage, and M. Rastegar, "Brain region-specific expression of MeCP2 isoforms correlates with DNA methylation within Mecp2 regulatory elements," PLoS One, vol. 9, no. 3, article e90645, 2014.

[92] R. M. Zachariah, C. O. Olson, C. Ezeonwuka, and M. Rastegar, "Novel MeCP2 isoform-specific antibody reveals the endogenous MeCP2E1 expression in murine brain, primary neurons and astrocytes," PLoS One, vol. 7, no. 11, article e49763, 2012. 
[93] S. Cohen, H. W. Gabel, M. Hemberg et al., "Genome-wide activity-dependent MeCP2 phosphorylation regulates nervous system development and function," Neuron, vol. 72, no. 1, pp. 72-85, 2011.

[94] M. Rutlin and S. B. Nelson, "MeCP2: phosphorylated locally, acting globally," Neuron, vol. 72, no. 1, pp. 3-5, 2011.

[95] K. Abdelmohsen, A. Lal, H. H. Kim, and M. Gorospe, "Posttranscriptional orchestration of an anti-apoptotic program by HuR," Cell Cycle, vol. 6, no. 11, pp. 1288-1292, 2014.

[96] A. Skliris, O. Papadaki, P. Kafasla et al., "Neuroprotection requires the functions of the RNA-binding protein HuR," Cell Death and Differentiation, vol. 22, no. 5, pp. 703-718, 2015.

[97] C. M. Brennan and J. A. Steitz", "HuR and mRNA stability," Cellular and Molecular Life Sciences, vol. 58, no. 2, pp. 266277, 2001.

[98] E. Hitti and K. S. A. Khabar, "Sequence variations affecting AU-rich element function and disease," Frontiers in Bioscience, vol. 17, no. 1, pp. 1846-1860, 2012.

[99] D. M. Tiruchinapalli, M. G. Caron, and J. D. Keene, "Activitydependent expression of ELAV/Hu RBPs and neuronal mRNAs in seizure and cocaine brain," Journal of Neurochemistry, vol. 107, no. 6, pp. 1529-1543, 2008.

[100] P. J. Uren, S. C. Burns, J. Ruan, K. K. Singh, A. D. Smith, and L. O. F. Penalva, "Genomic analyses of the RNA-binding protein $\mathrm{Hu}$ antigen $\mathrm{R}(\mathrm{HuR}$ ) identify a complex network of target genes and novel characteristics of its binding sites," The Journal of Biological Chemistry, vol. 286, no. 43, pp. 37063-37066, 2011.

[101] X. Yang, X. Sun, J. Wu et al., "Regulation of the SIRT1 signaling pathway in NMDA-induced excitotoxicity," Toxicology Letters, vol. 322, pp. 66-76, 2020.

[102] N. Craddock, M. C. O'Donovan, and M. J. Owen, "Phenotypic and genetic complexity of psychosis," The British Journal of Psychiatry, vol. 190, no. 3, pp. 200-203, 2007.

[103] J. M. McClellan, E. Susser, and M. C. King, "Schizophrenia: a common disease caused by multiple rare alleles," The British Journal of Psychiatry, vol. 190, no. 3, pp. 194-199, 2007.

[104] T. Walsh, J. M. McClellan, S. E. McCarthy et al., "Rare structural variants disrupt multiple genes in neurodevelopmental pathways in schizophrenia," Science, vol. 320, no. 5875, pp. 539-543, 2008.

[105] A. Dewulf, Anatomy of the Normal Human Thalamus: Topometry and Standardized Nomenclature, Elsevier, Amsterdam, 1971. 